\title{
Copeptin level in the early prediction of cardiorenal syndrome in rats
}

\author{
XIAOSHENG SHENG ${ }^{1}$, LI LIN ${ }^{1}$, FANGMING GUO ${ }^{2}$, SHUXIA LIANG ${ }^{3}$, \\ HAOHAO CHEN ${ }^{2}$, YUANSHU FANG ${ }^{4}$ and MINGXING DING ${ }^{2}$
}

\author{
${ }^{1}$ Department of Cardiology, Jinhua People's Hospital, Jinhua, Zhejiang 321000; ${ }^{2}$ Department of \\ Medical Sciences, Jinhua Polytechnic, Jinhua, Zhejiang $321007 ;{ }^{3}$ Endoscopy Center, \\ Jinhua Hospital of Zhejiang University, Jinhua, Zhejiang 321001; ${ }^{4}$ Department of Laboratory Animals Center, \\ Jinhua Institute for Food and Drug Control, Jinhua, Zhejiang 321000, P.R. China
}

Received July 5, 2017; Accepted May 24, 2018

DOI: $10.3892 /$ etm.2018.6239

\begin{abstract}
Copeptin (CPP) has been considered as a useful marker for prediction of prognosis in heart diseases. However, CPP has not been investigated sufficiently in cardiorenal syndrome (CRS). The present study aimed to investigate the value of CPP level in predicting CRS in rats with partial nephrectomy combined with myocardial infarction $(\mathrm{SNX}+\mathrm{MI})$. A total of 60 male Sprague-Dawley rats were used to establish the CRS model by partial nephrectomy combined with MI. The rats were randomly divided into blank control (CK), SNX, MI and CRS groups. Changes in serum and urine CPP concentrations, hemodynamics, blood pressure, and renal function were examined 1-5 weeks after modeling. The predictive values of CPP in the occurrence of CRS in rats were evaluated using receiver operating characteristic (ROC) curve. The results showed that serum CPP in the CRS group in 1-5 weeks and urine CPP in 3 weeks after modeling increased significantly compared with the CK group. Also, serum B-type natriuretic peptide (BNP) in 1 and 3 weeks and urine BNP in 4-5 weeks after modeling increased significantly. No correlation was found between serum or urine CPP, BNP and BUN levels 1 week after modeling in the CRS group. The ROC curve analysis showed that the area under the curve of CRS predicted by serum CPP at 1 week was 0.908 with $56.59 \mathrm{pg} / \mathrm{ml}$ as the cutoff point, and its diagnostic sensitivity and specificity were 87.5 and $80.0 \%$, respectively. To conclude, SNX + MI may be used to establish CRS rat model with cardiac and renal damage. Serum CPP may serve as a specific biomarker for the early prediction of CRS.
\end{abstract}

Correspondence to: Professor Mingxing Ding, Department of Medical Sciences, Jinhua Polytechnic, 1188 Wuzhou Road, Jinhua, Zhejiang 321007, P.R. China

E-mail: mxding@hotmail.com

Key words: copeptin, B-type natriuretic peptide, cardiorenal syndrome, rats

\section{Introduction}

Cardiorenal syndrome (CRS) refers to the clinical syndrome when the patients suffer from cardiac insufficiency and renal impairment that have a complex interaction (1). CRS includes different pathophysiological disorders of the heart and the kidney. Furthermore, acute and chronic dysfunction of any organ would lead to acute and chronic dysfunction of another, both of which are clinical risk factors for each other. Their mutual aggravation leads to rapid deterioration of cardiac and renal functions to increase mortality. Therefore, it is now necessary for us to expand our knowledge regarding its pathogenesis, prevention, and potential treatment, and it is extremely urgent to seek early specific and sensitive biomarkers to predict cardiac and renal damage (2).

Biomarkers may improve risk predictive models in the future. Biomarkers pertinent to the cardiovascular $(\mathrm{CV})$ and renal interface are defined as renocardiovascular biomarkers and currently the emerging biomarkers such as B-type natriuretic peptide (BNP), cardiac troponins, copeptin (CPP), the biomarker of renal injury (neutrophil gelatinase-associated lipocalin) have been introduced (3). For example, CPP recently has been demonstrated to be potential suitable for guiding management of patients with cardiac and renal damage. CPP is part of the original $\mathrm{C}$-terminal of arginine vasopressin (AVP), which is stable in vivo and easy to be detected. AVP is an effective osmoregulator that can increase peripheral vasoconstrictive activity through interaction with its receptor V1. On the other hand, binding to the V2 receptor mediates water retention in renal tubules (4). Unfortunately, the circulatory half-life of AVP is very short rendering it inaccessible for clinical routine determination. CPP has been found to have an important clinical value in the early diagnosis and prognosis of $\mathrm{CV}$ and cerebrovascular diseases such as acute MI $(3,5)$. In addition, it could also be useful in the diagnosis of left ventricular dysfunction (LV dysfunction) in hemodialysis patients (6). However, few studies are related to the occurrence of CRS as well as its diagnosis and treatment, leading to the lack of animal model data, although CPP has been found to be associated with renal function in chronic kidney disease (CKD) (7). Therefore, this study used the combination of 
partial nephrectomy and myocardial infarction $(\mathrm{SNX}+\mathrm{MI})$ to aggravate organ damage for establishing CRS rat model to observe the dynamic changes in serum and urine CPP concentrations. Moreover, a comparative analysis was performed with indicators such as BNP to evaluate the predictive value of CPP level in CRS rats, which provided a basis for seeking new markers for the diagnosis and prognosis of CRS.

\section{Materials and methods}

Animal modeling and grouping. A total of 60 specific pathogen-free male Sprague-Dawley (SD) rats weighed 250-280 g were purchased from Shanghai Laboratory Animal Center Laboratory Animal Co., Ltd. The license number was SCXK (Shanghai) 2012-0002, and the certificate number was 0278317. After the experimental animals were adapted in the animal room for 6 days, they were divided into 4 groups (15 rats in each group) using random selection (the animals were numbered, and 2 rats were randomly selected from each brood to be placed in the same group), including CK group (blank control group), SNX group (renal failure group), MI group (heart failure group), and CRS group (heart and renal failure group). Modeling was performed using combined surgery according to the literature (8).

The blank control group (CK group): The rats in this group were routinely fed without any other treatment.

The SNX group: The rats in this group were abdominally anesthetized using $10 \%$ chloral hydrate $(300 \mathrm{mg} / \mathrm{kg}$, i.p.; Qingxi Chemical Technology Co., Ltd., Shanghai, China) and fixed in a supine position. The fur of the abdomen was removed, and disinfection was performed using iodophor. The abdominal cavity was opened to ligate renal artery of the left kidney, renal vein, and ureter, and then the left kidney was excised. The abdominal cavity was closed, and suturing was performed layer by layer. The animals were placed back into the cage after they awoke, and 100,000 units of penicillin sodium were intramuscularly injected for anti-infection 3 days after the surgery.

The MI group: The rats in this group were abdominally anesthetized using $10 \%$ chloral hydrate $(300 \mathrm{mg} / \mathrm{kg}$, i.p., Qingxi Chemical Technology Co., Ltd.) and fixed in a supine position. The fur of the chest was removed, and disinfection was performed using iodophor. Electrocardiogram (ECG) was monitored using limb II lead. The rats wore breathing masks (made by researchers) on the mouth and nose, which were connected to the ventilator to maintain positive end-expiratory pressure (parameters of the ventilator were set as follows: Respiratory rate of 55-60 times/min, breathing ratio of 1:2, and tidal volume of $20 \mathrm{ml} / \mathrm{kg}$ ). The skin of the left chest was cut off, and the muscle was bluntly separated. The intercostal opener was used to stretch ribs in third and fourth intercostal spaces, and the cardiac pericardium was torn open to expose the heart. A needle was injected 2-3 $\mathrm{mm}$ below the lower edge of the left atrial appendage using great cardiac vein between the left atrial appendage and the pulmonary artery cone as a marker, and the needle was removed from the pulmonary artery cone. The depth of the injection was $1.5-2 \mathrm{~mm}$, and the width was $2-3 \mathrm{~mm}$. The left coronary artery was ligated. It was visually observed that the left ventricular wall was whitened, ECG QRS wave was heighted and broadened, and the intersection ( $\mathrm{J}$ point) of the $\mathrm{S}$ wave and $\mathrm{T}$ wave elevated significantly, or the $\mathrm{R}$ wave disappeared. The ligation was successful when a typical QS wave occurred. Then, the chest was closed, and the muscle and skin were sutured layer by layer. An intravenous catheter system was indwelled before closing the chest to extract air from the chest cavity to restore negative pressure. The animals were placed back into the cage to be fed after they awoke. Next, 100,000 units of penicillin sodium was intramuscularly injected for anti-infection 3 days after the surgery.

The CRS group: The left kidney of rats in this group was excised, and the coronary artery was ligated to perform modeling.

This study was approved by the Animal Ethics Committee of the JinHua Center of Laboratory Animals. All experimental procedures were carried out in accordance with the approval agreement of experimental animals.

Evaluation of cardiac function and blood pressure (BP). According to the literature, cardiac function and BP were detected (9). At week 5 before sacrifice, the rats in each group were abdominally anesthetized using $10 \%$ chloral hydrate (300 mg/kg, i.p.; Qingxi Chemical Technology Co., Ltd.). Then, a 2-cm incision was made longitudinally from the middle of the neck to expose the right common carotid artery. After that, a PE50 catheter was inserted into the left ventricle and cardiac function parameters were analyzed using a biological signal processing system (Techman Soft, Chengdu, China; model no. BL-420S), including changes in heart rate (HR), left ventricular systolic blood pressure (LVSP), left ventricular end diastolic pressure (LVEDP), and $\pm \mathrm{dp} / \mathrm{dt}$ max (change rate of left ventricular pressure). BP changes of rats in each group were measured using a tail vein noninvasive BP measurement system (Techman Soft; model no. BP-300). The measurement was performed once every other week for a total of five times.

Evaluation of renal function. After successful modeling, the animals were anesthetized with $10 \%$ chloral hydrate (300 mg/kg, i.p.,Qingxi Chemical Technology Co.,Ltd.) prior to eye blood collection, then blood was extracted from the orbital cavity and urine was collected, once every week for a total of five times, and then renal function was tested. The serum BUN level in each group was measured by a biochemical method using a BUN test box (Nanjing Jiancheng Bioengineering Institute; item no. C013-2; batch no. 20161228), according to the manufacturer's instructions. Serum and urine $\mathrm{Cr}$ levels of each group were measured by the sarcosine oxidase enzymatic method using a creatine kinase assay kit (Reebio, Ningbo, China; item no. 2400746; batch no. 2017020802), according to the manufacturer's instructions also (10).

Measurement of CPP and BNP levels using enzyme-linked immunosorbent assay. CPP and BNP levels were assayed using enzyme-linked immunosorbent assay (ELISA) in accordance with the instructions from the kits provided (11). After successful modeling, blood was extracted from the orbital cavity and urine was collected once every week for a total of five times. ELISA kits for CPP and BNP [ELISA kit for CPP was purchased from USCN Life Science, Inc., (Wuhan, China), item no. SEA365Ra and batch no. 161101216; ELISA kit for 

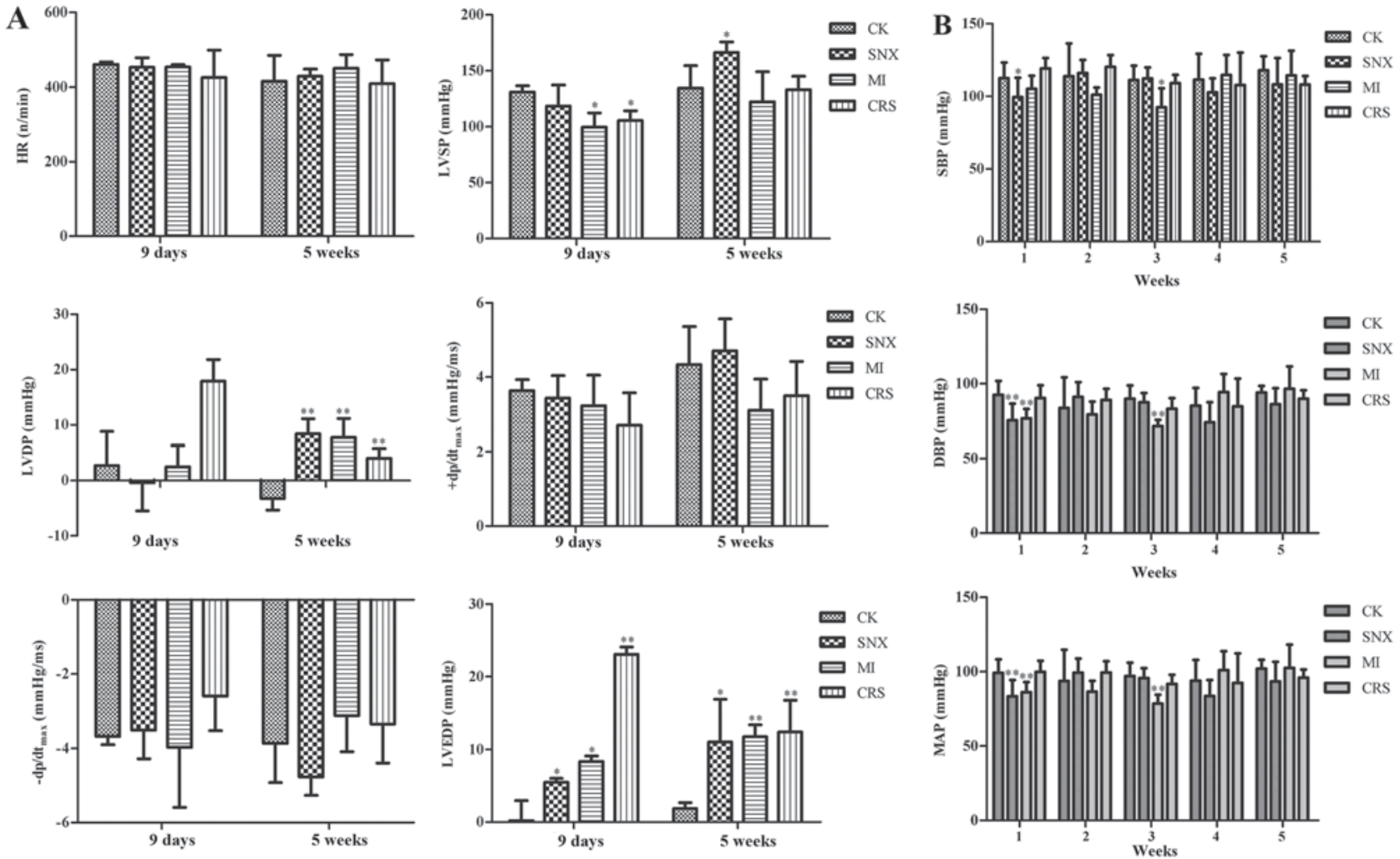

Figure 1. Changes in hemodynamics (A) and BP (B) in each group. ${ }^{*} \mathrm{P}<0.05,{ }^{* *} \mathrm{P}<0.01$, compared with the CK group. HR, heart rate; LVSP, left ventricular systolic blood pressure; LVEDP, left ventricular end diastolic pressure; $\pm \mathrm{dp} / \mathrm{dt}$ max, change rate of left ventricular pressure. SBP, systolic blood pressure; DBP, diastolic blood pressure; MAP, mean arterial pressure.

BNP with the item no. CEA541Ra and batch no. 161101217] were used to measure serum and urine CPP together with BNP levels strictly based on the kit instruction.

Histopathological observation of heart and kidney. The rats were sacrificed at day 9 and week 5 after modeling. Then, conventional hematoxylin and eosin (H\&E) staining was performed for cardiac and renal tissues, and the tissue slices were examined under a light microscope to determine pathological changes in cardiac and renal tissues in CRS rats.

Statistical analysis. All data were analyzed using the SPSS22.0 statistical software package (SPSS, Inc., Chicago, IL, USA). Continuous data were represented by mean \pm standard deviation. The intragroup comparison was done using the independent-sample t-test or one-way analysis of variance, further comparison between the groups was performed using the least significant difference t-test, and non-normal distribution data was analyzed using the nonparametric test. The correlation analysis of normal distribution data was performed using Pearson linear correction, whereas non-normal distribution data was analyzed using Spearman rank correlation. Receiver operating characteristic (ROC) curve was used to determine the early diagnostic value of CPP, BNP, and BUN on CRS. $\mathrm{P}<0.05$ was considered to indicate a statistically significant difference.

\section{Results}

General condition and survival rate. After 5 weeks, the rats in each modeling group showed deeper color of fur, less appetite, no increase in weight and exercise, and mouth and nose in cyan color compared with the control group. Asthma and edema in the feet occurred. Moreover, prehension response was weakened. In the MI group, one rat died within 5 weeks, and the survival rate was $85.7 \%$. In the CRS group, two rats died within 5 weeks, and the survival rate was $71.4 \%$. No death occurred in the CK and SNX groups, and the survival rate was $100 \%$.

Cardiac function and BP changes in each group. The results of hemodynamic test indicated that LVEDP in the SNX group increased significantly in 9 days compared with CK group $(\mathrm{P}<0.05)$. LVSP in the MI group decreased significantly in 9 days $(\mathrm{P}<0.05)$, and LVEDP increased significantly $(\mathrm{P}<0.05)$. LVSP in the CRS group decreased significantly in 9 days $(\mathrm{P}<0.05)$, and LVEDP increased significantly $(\mathrm{P}<0.01)$. The results of BP estimation demonstrated that systolic BP (SBP), diastolic BP (DBP) and MAP (mean pressure) in the SNX group decreased significantly in 1 week compared with the CK group $(\mathrm{P}<0.05)$. SBP, DBP, and MAP in the MI group decreased significantly in 3 weeks $(\mathrm{P}<0.05, \mathrm{P}<0.01$, and $\mathrm{P}<0.01$, respectively), whereas no statistically significant difference in SBP, DBP, and MAP was observed in the CRS group at each time point $(\mathrm{P}>0.05$; Fig. 1$)$.

Renal function changes in each group. The results showed that compared with the CK group, serum BUN in the MI group elevated significantly in 5 weeks after modeling $(\mathrm{P}<0.05)$, serum BUN in the SNX group elevated significantly in 3-5 weeks after modeling $(\mathrm{P}<0.01)$, serum BUN in the CRS 

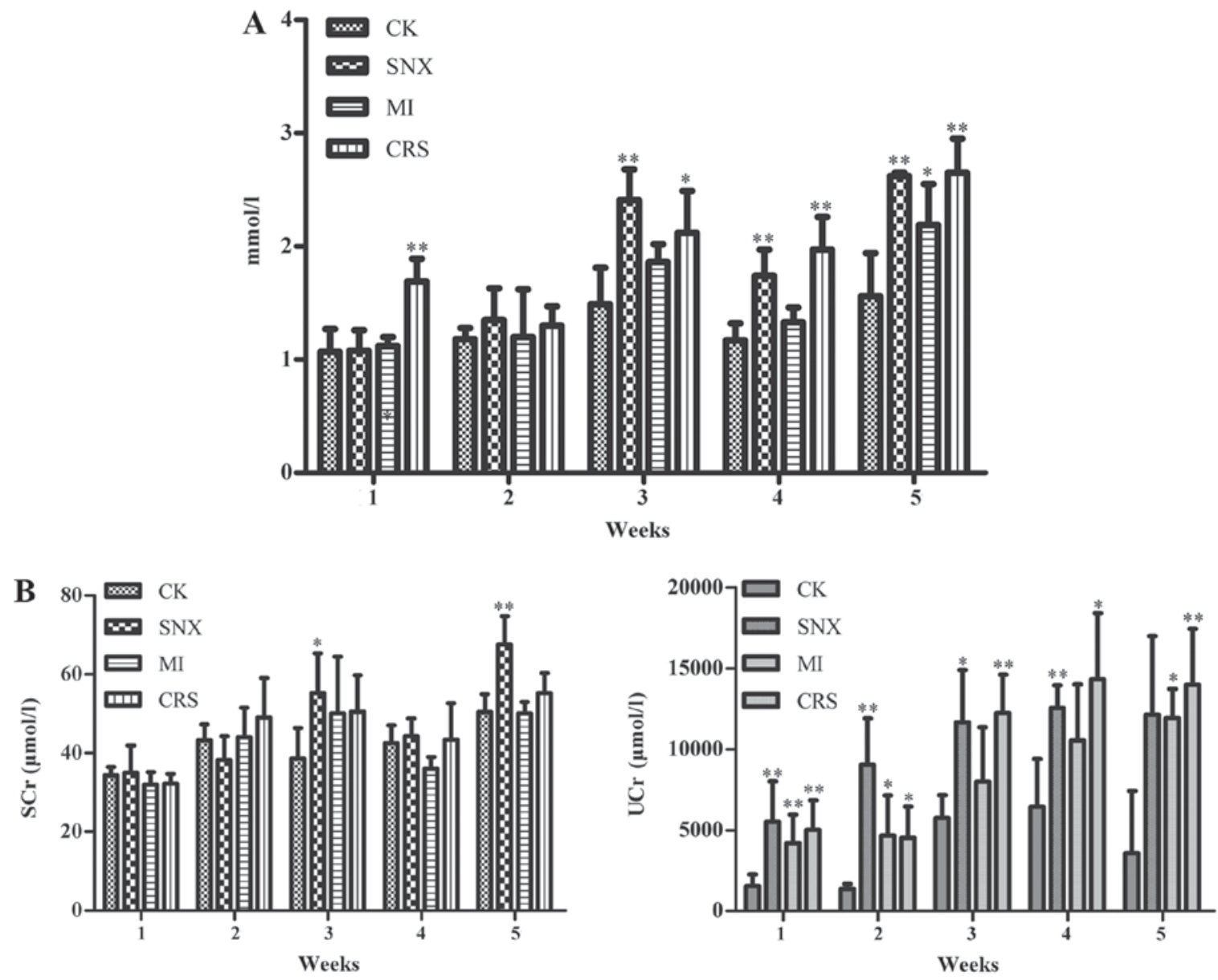

Figure 2. Renal function changes in each group in 1-5 weeks. (A) serum BUN. (B) $\mathrm{SCr}$ and $\mathrm{UCr}$. ${ }^{*} \mathrm{P}<0.05,{ }^{* *} \mathrm{P}<0.01$, compared with the CK group. UCr, urine creatinine; $\mathrm{SCr}$, serum creatinine; BUN, blood urea nitrogen.

group elevated significantly in 1 week and 3-5 weeks after modeling $(\mathrm{P}<0.01, \mathrm{P}<0.05)$. $\mathrm{SCr}$ in the $\mathrm{SNX}$ group increased significantly in 3 weeks and 5 weeks $(\mathrm{P}<0.05)$, and $\mathrm{UCr}$ increased significantly in $1-4$ weeks $(\mathrm{P}<0.01, \mathrm{P}<0.05)$. $\mathrm{UCr}$ in the MI group increased significantly in 1-2 weeks and 5 weeks $(\mathrm{P}<0.01, \mathrm{P}<0.05) . \mathrm{UCr}$ in the CRS group increased significantly in $1-5$ weeks $(\mathrm{P}<0.01$; Fig. 2).

Changes in CPP and BNP levels as well as their relationship with other indicators. Serum CPP in the SNX group increased significantly in 2-5 weeks after modeling compared with the CK group $(\mathrm{P}<0.05, \mathrm{P}<0.01)$, and urine CPP increased significantly in $2-3$ weeks and 5 weeks after modeling $(\mathrm{P}<0.05$, $\mathrm{P}<0.01)$. Serum CPP in the MI group increased significantly in $2-4$ weeks after modeling $(\mathrm{P}<0.05, \mathrm{P}<0.01)$. Serum $\mathrm{CPP}$ in the CRS group increased significantly in $1-5$ weeks after modeling $(\mathrm{P}<0.05, \mathrm{P}<0.01)$, and urine $\mathrm{CPP}$ increased significantly in 3 weeks after modeling $(\mathrm{P}<0.05)$. Meanwhile, compared with the CK group, serum BNP in the SNX group increased significantly in $1-3$ weeks after modeling $(\mathrm{P}<0.05, \mathrm{P}<0.01)$, while urine BNP increased significantly in 4-5 weeks after modeling $(\mathrm{P}<0.01)$. Urine $\mathrm{BNP}$ in the MI group increased significantly in 1 week, 3-5 weeks after modeling $(\mathrm{P}<0.05, \mathrm{P}<0.01)$. Serum BNP in the CRS group increased significantly in 1 week and 3 weeks after modeling $(\mathrm{P}<0.01)$, while urine BNP increased significantly in 4-5 weeks after modeling ( $\mathrm{P}<0.05$; Fig. 3 ).
The correlation analysis demonstrated that serum CPP, BNP, and BUN in the CRS group in 1 week after modeling were not correlated $(r=0.109$ and 0.683 , respectively; $\mathrm{P}>0.05)$. Moreover, urine CPP, BNP, and BUN were also not correlated $(r=-0.342$ and 0.293 , respectively; $\mathrm{P}>0.05)$.

$C P P$ and BNP levels in the early diagnosis of CRS. Test results of serum CPP (sCPP), urine CPP (uCPP), and BNP in 1 week and 3 weeks in each model group were selected as the test variables to diagnose CRS, and the groups were used as the state variables. Moreover, SNX and MI groups were defined as 0 ; CRS group was defined as 1; and ROC curve analysis was performed. The results revealed that the area under the curve (AUC) of sCPP in 1 week was 0.908 (95\% CI: 0.789-1.028). With $56.59 \mathrm{pg} / \mathrm{ml}$ as the cutoff point, the diagnostic sensitivity was $87.5 \%$ and the specificity was $80.0 \%$. However, the test results of sCPP in 3 weeks and $\mathrm{uCPP}$ and BNP in 1 week and 3 weeks were not statistically significant $(P>0.05$; (Fig. 4 and Table I).

Pathological changes in cardiac and renal tissues in each group. H\&E staining of cardiac tissue showed no significant pathological changes in the CK group, while SNX, MI, and CRS groups showed myocardial tissue disorder, some myocardial degeneration, and dissolution and rupture together with congestion and expansion, which were significant in the CRS 

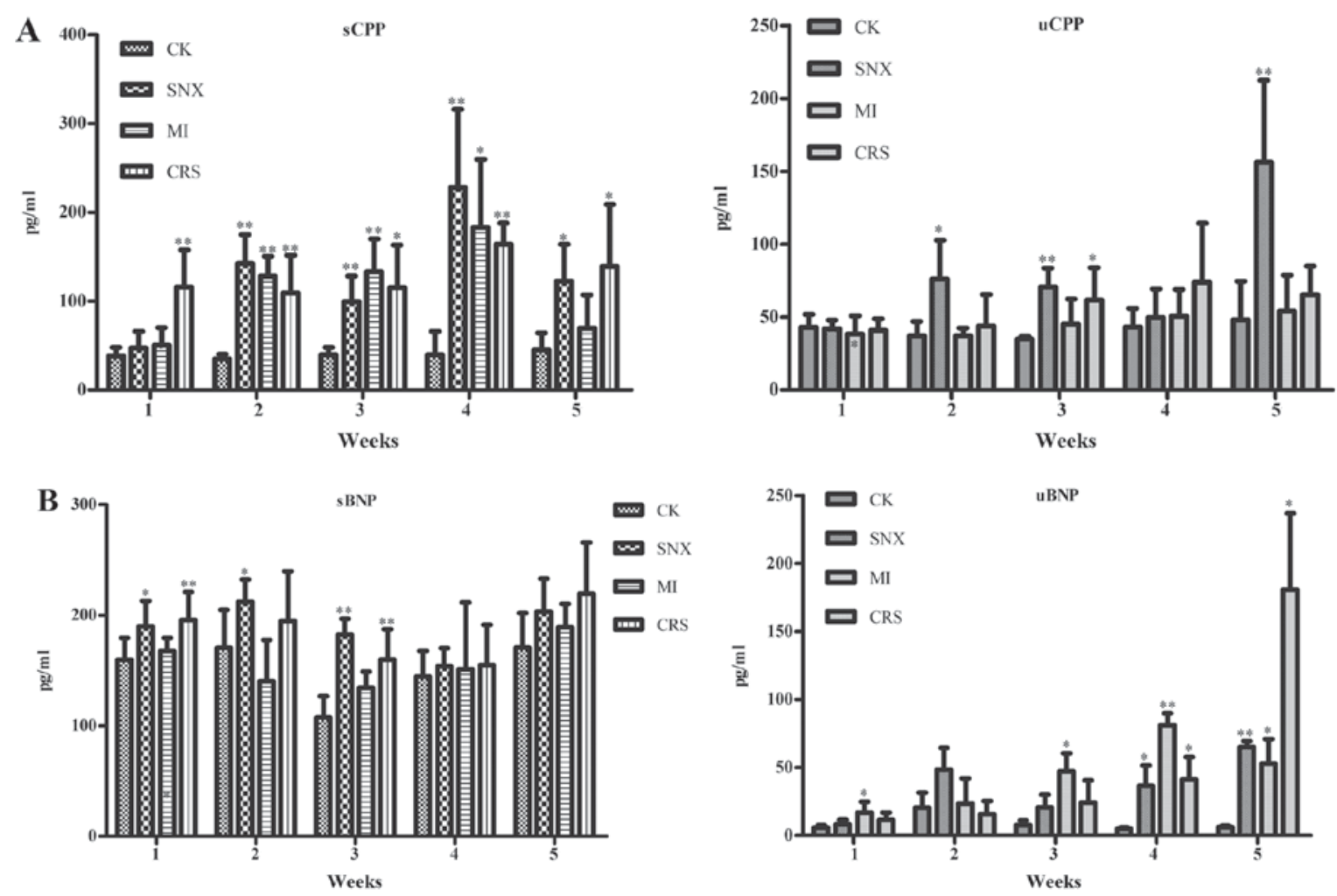

Figure 3. CPP and BNP levels in each group in $1-5$ weeks. (A) sBNP and uBNP. (B) CPP. ${ }^{*} \mathrm{P}<0.05,{ }^{* *} \mathrm{P}<0.01$, compared with the CK group. sBNP, serum B-type natriuretic peptide; $\mathrm{uBNP}$, urine B-type natriuretic peptide; sCPP, serum copeptin.

group (Fig. 5A, C, E and G). H\&E staining of renal tissue demonstrated that the CK group had no significant pathological change; however, the SNX and MI groups showed mild injury, and the CRS group was the most serious (Fig. 5B, D, $\mathrm{F}$ and $\mathrm{H}$ ).

\section{Discussion}

This study confirmed that the combination of SNX + MI aggravated organ damage to establish a CRS rat model, which could reflect the two-way interaction of heart and kidney and could be used to evaluate the diagnostic effect of CRS. It had been confirmed by dynamically observing changes in serum and urine CPP concentrations 1-5 weeks after modeling that the detection of the serum CPP level of CRS rats was conducive to early detection of cardiac and renal damage, which displayed a warning effect. Moreover, CPP could be used as a potential biomarker for the early prediction of CRS with high sensitivity and specificity.

The relationship between cardiac diseases and renal diseases has been widely explored with the proposal of CRS concept in recent years. A stable and reliable animal model is an urgent need for further clinical studies. The CRS rat model was first introduced in 2004, and myocardial infarction combined with unilateral nephrectomy (UNX + MI) was used, but this model did not find any progressive impairment of cardiac function combined with renal function degeneration (12). However, the SNX + MI method used in this study was designed by Entin-Meer et al (13) in the study of CRS model, which demonstrated its advantages by partial nephrectomy combined with MI, indicating the two-way interaction of heart and kidney. This study found, through the dynamical observation of hemodynamics, BP, and renal function 1-5 weeks after modeling, that the degeneration of cardiac and renal function occurred 1 week after modeling, suggesting that rats developed cardiac and renal damage in a short time. Thus, this model could highly stimulate the angiographic features of clinical CRS, providing experimental methods for exploring the pathophysiological mechanism and for drug research of CRS. The myocardium of some rats with renal injury due to nephrectomy was more prone to ischemic injury, and the MI area of uremic rats increased significantly. These findings were consistent with the finding that the mortality rate of patients with renal diseases combined with MI was higher. Due to partial nephrectomy, erythropoietin secreted by kidney decreased, blood oxygen capacity decreased, cardiac ejection aggravated, blood return of heart and various organs increased, vessels contracted, cardiac hypertrophy aggravated, and BP increased. Moreover, endothelial damage led to a decrease in nutritional function of the coronary artery, aggravating myocardial ischemia. Meanwhile, cardiac dysfunction could, in turn, accelerate the inherent damage of kidney (14).

The occurrence of CRS is accompanied by the corresponding changes in different biomarkers, which plays an important role in the early diagnosis, risk assessment, and prognosis. Due to capacity load and sodium retention as well as the inappropriate activation of atrial natriuretic peptide, AVP, and renin-angiotensin system, water metabolism disorder caused by CRS is more prominent. Among them, the AVP system may be another neuroendocrine system closely related to the poor prognosis of acute myocardial infarction (AMI) or cardiac failure (15). The CPP test is easy and 
Table I. ROC curve analysis of sCPP, uCPP and BNP in the diagnosis of cardiorenal syndrome.

\begin{tabular}{|c|c|c|c|c|c|c|c|}
\hline Indicators & AUC & SE & P-value & $95 \% \mathrm{CI}$ & Cutoff value & Sensitivity & Specificity \\
\hline \multicolumn{8}{|l|}{ sCPP } \\
\hline 1 week & 0.908 & 0.061 & 0.002 & $0.789-1.028$ & 56.590 & 0.875 & 0.800 \\
\hline 3 weeks & 0.489 & 0.181 & 0.947 & $0.134-0.844$ & 84.455 & 0.600 & 0.444 \\
\hline \multicolumn{8}{|l|}{ uCPP } \\
\hline 1 week & 0.475 & 0.130 & 0.846 & $0.221-0.729$ & 45.815 & 0.625 & 0.533 \\
\hline 3 weeks & 0.422 & 0.173 & 0.641 & $0.083-0.761$ & 60.665 & 0.400 & 0.444 \\
\hline \multicolumn{8}{|l|}{ BNP } \\
\hline 1 week & 0.617 & 0.126 & 0.366 & $0.370-0.863$ & 181.250 & 0.625 & 0.667 \\
\hline 3 weeks & 0.489 & 0.164 & 0.947 & $0.168-0.810$ & 167.480 & 0.600 & 0.556 \\
\hline
\end{tabular}

ROC, receiver operating characteristic; sCPP, serum copeptin; uCPP, urine copeptin; BNP, B-type natriuretic peptide; CI, confidence interval; AUC, area under the curve.
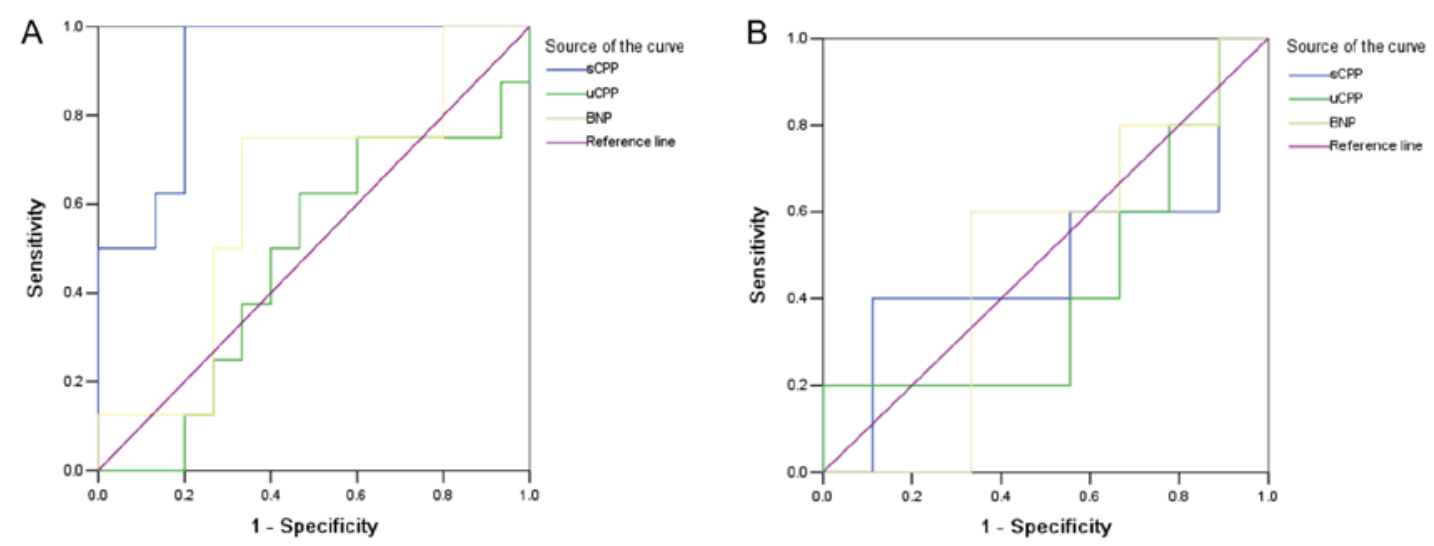

Figure 4. ROC curve of sCPP, uCPP and BNP in the diagnosis of CRS. (A) In 1 week. (B) In 3 weeks. sCPP, serum copeptin; uCPP, urine copeptin; BNP, B-type natriuretic peptide.

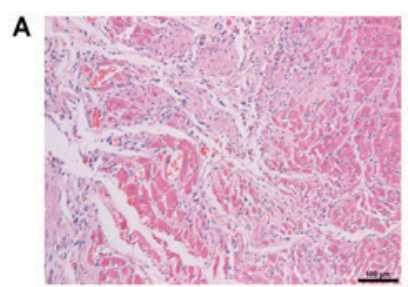

E

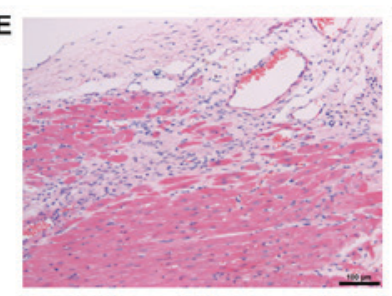

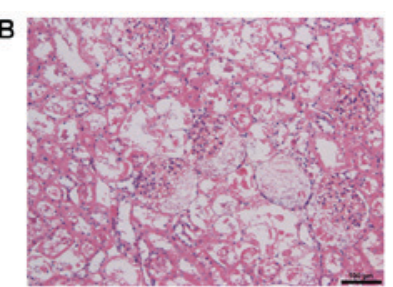

$\mathbf{F}$

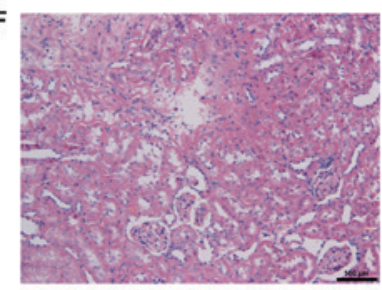

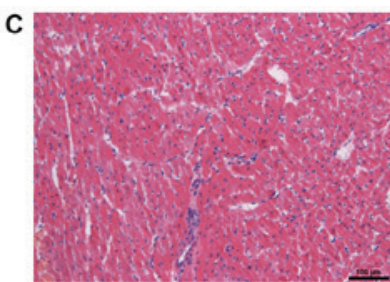
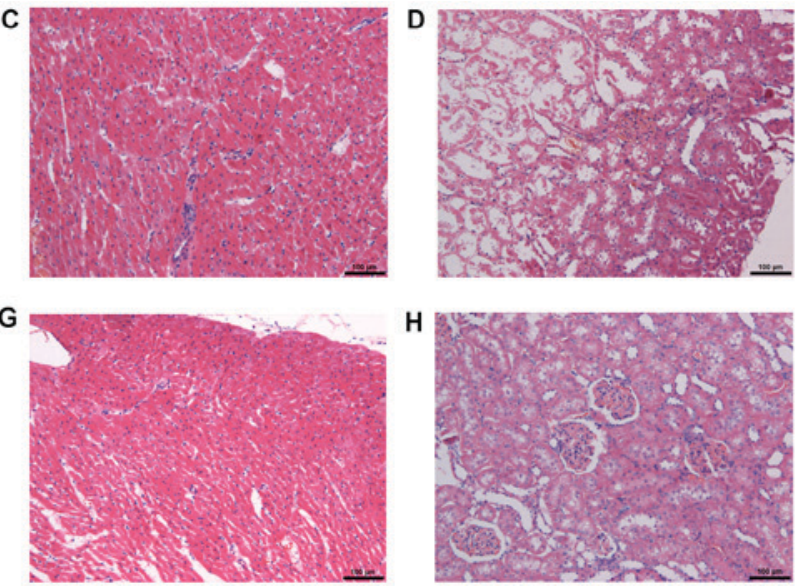

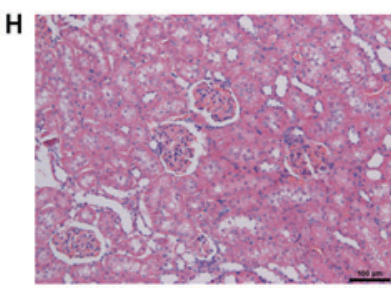

Figure 5. Pathological changes in cardiac and renal tissues in each group. The pathological changes in H\&E-stained myocardial tissue sections in the CRS group (A), SNK group (C), MI group (E), and CK group (G). The pathological changes in H\&E-stained renal tissue sections in the CRS group (B), SNK group (D), MI group (F), and CK group (H).

convenient, and its results are stable, which can be carried out at all levels of hospitals. Moreover, the CPP test can replace the direct measurement of AVP. This study dynamically detected changes in serum and urine CPP concentrations
1-5 weeks after modeling and found that serum and urine CPP in 1 week and 3 weeks after modeling increased significantly in the CRS group, and only serum CPP increased in 5 weeks after modeling. The ROC curve analysis showed that serum 
CPP in 1 week after modeling had higher specificity (80\%) and sensitivity (87\%) in predicting CRS, suggesting that CPP changed in the early stage of cardiac and renal damage, but it tended to be stable in the late stage, which was expected to provide a new and good biomarker for the early diagnosis of CRS. Therefore, CPP had a broad application prospect. In fact, CPP has recently been proved to have a predictive value in multiple clinical diseases, including $\mathrm{CV}$ diseases, such as heart failure, myocardial infarction, and resuscitation $(6,16,17)$. However, few reports are available on kidney-related diseases. Only Kim et al (6) confirmed that CPP could be a useful indicator for diagnosing left ventricular dysfunction in patients receiving hemodialysis. The ROC analysis showed that AUC was 0.737 , the cutoff point was $125.48 \mathrm{pg} / \mathrm{ml}$, and the sensitivity and specificity were 0.7 and 0.8 , respectively. However, it was believed that the potential to predict the risk of $\mathrm{CV}$ events in perioperative $\mathrm{CV}$ surgery for patients with chronic kidney disease was insufficient (18), or in neonatal and perinatal asphyxia animal models, CPP was not a specific marker for diagnosing acute renal injury (19). Further correlation analysis of this study showed that serum or urine CPP, BNP, and BUN were not correlated, which might be related to the complex pathophysiology of CRS. BNP was publicly recognized as a clinical diagnostic marker of heart failure. Although BNP was reported to have a certain application value in the early diagnosis of CRS $(2,20,21)$, whether it would accumulate in the body of patients with CRS due to heart failure or the decrease in glomerular filtration rate, as well as its diagnostic value for renal insufficiency, still needed to be further studied (22).

CRS is a complex clinical syndrome with various difficulties in its treatment due to the uncertainty of its definition and pathophysiological mechanism. Early diagnosis is one of the most effective ways to improve its efficacy, and the development of new biomarkers is the key to early diagnosis. In conclution, present study confirmed that the combination of SNX + MI could be used to establish a CRS rat model with cardiac and renal injury. Moreover, CPP could be used as a potentially sensitive and specific biomarker for the early diagnosis of CRS.

\section{Acknowledgments}

Not applicable.

\section{Funding}

This study was supported by Experimental Animals Science and Technology Project of Zhejiang Province in China (grant no. 2015C37088).

\section{Availability of data and materials}

All data generated or analyzed during this study are included in this published article.

\section{Authors' contributions}

XS, LL and MD conceived and coordinated the study, designed, performed and analyzed the experiments, and wrote the paper. FG and SL carried out the data collection and data analysis. HC and YF performed and analyzed the experiments. All authors reviewed the results and approved the final version of the manuscript.

\section{Ethics approval and consent to participate}

This study was approved by the Animal Ethics Committee of the JinHua Center of Laboratory Animals.

\section{Consent for publication}

Not applicable.

\section{Competing interests}

The authors declare that they have no competing interests.

\section{References}

1. Bongartz LG, Braam B, Gaillard CA, Cramer MJ, Goldschmeding R, Verhaar MC, Doevendans PA and Joles JA: Target organ cross talk in cardiorenal syndrome: Animal models. Am J Physiol Renal Physiol 303: F1253-F1263, 2012.

2. Hou FF and Yang X: Advances in the management of acute cardiorenal syndrome in China: Biomarkers for predicting development and outcomes. Kidney Dis (Basel) 2: 145-150, 2017.

3. Niizuma S, Iwanaga $Y$, Yahata $T$ and Miyazaki $S$ : Renocardiovascular biomarkers: From the perspective of managing chronic kidney disease and cardiovascular disease. Front Cardiovasc Med 4: 10, 2017.

4. Morgenthaler NG, Struck J, Jochberger S and Dünser MW: Copeptin: Clinical use of a new biomarker. Trends Endocrinol Metab 19: 43-49, 2008.

5. Morawiec B and Kawecki D: Copeptin: A new marker in cardiology. J Cardiovasc Med (Hagerstown) 14: 19-25, 2013.

6. Kim JS, Yang JW, Chai MH, Lee JY, Park H, Kim Y, Choi SO and Han BG: Copeptin in hemodialysis patients with left ventricular dysfunction. Yonsei Med J 56: 976-980, 2015.

7. Velho G, Bouby N, Hadjadj S, Matallah N, Mohammedi K, Fumeron F, Potier L, Bellili-Munoz N, Taveau C, Alhenc-Gelas F, et al: Plasma copeptin and renal outcomes in patients with type 2 diabetes and albuminuria. Diabetes Care 36: 3639-3645, 2013.

8. Windt WA, Henning RH, Kluppel AC, Xu Y, de Zeeuw D and van Dokkum RP: Myocardial infarction does not further impair renal damage in 5/6 nephrectomized rats. Nephrol Dial Transplant 23: 3103-3110, 2008.

9. Peng DF, Tang SY, Hu YJ, Chen J and Yang L: Pathophysiological model of chronic heart failure complicated with renal failure caused by three-quarter nephrectomy and subcutaneous injection of isoprenaline. Exp Ther Med 5: 835-839, 2013.

10. Pang L, Lian X, Li Y, Nan L and Ma H: Efficacy and safety of parecoxib sodium after renal transplantation. African J Pharm Pharmacol 5: 2467-2473, 2011.

11. Aydin S, Eren MN, Kuloglu T, Aydin S, Yilmaz M, Gul E, Kalayci M, Yel Y, Cakmak T and Bico S: Alteration of serum and cardiac tissue adropin, copeptin, irisin and TRPM2 expressions in DOX treated male rats. Biotech Histochem 90: 197-205, 2015.

12. van Dokkum RP, Eijkelkamp WB, Kluppel AC, Henning RH, van Goor H, Citgez M, Windt WA, van Veldhuisen DJ, de Graeff PA and de Zeeuw D: Myocardial infarction enhances progressive renal damage in an experimental model for cardio-renal interaction. J Am Soc Nephrol 15: 3103-3110, 2004.

13. Entin-Meer M, Ben-Shoshan J, Maysel-Auslender S, Levy R, Goryainov P, Schwartz I, Barshack I, Avivi C, Sharir R and Keren G: Accelerated renal fibrosis in cardiorenal syndrome is associated with long-term increase in urine neutrophil gelatinase-associated lipocalin levels. Am J Nephrol 36: 190-200, 2012.

14. Liu S, Kompa AR, Kumfu S, Nishijima F, Kelly DJ, Krum H and Wang BH: Subtotal nephrectomy accelerates pathological cardiac remodeling post-myocardial infarction: implications for cardiorenal syndrome. Int J Cardiol 168: 1866-1880, 2013. 
15. Lipinski MJ, Escárcega RO, D'Ascenzo F, Magalhães MA, Baker NC, Torguson R, Chen F, Epstein SE, Miró O, Llorens $\mathrm{P}$, et al: A systematic review and collaborative meta-analysis to determine the incremental value of copeptin for rapid rule-out of acute myocardial infarction. Am J Cardiol 113 : $1581-1591,2014$

16. Boeckel JN, Oppermann J, Anadol R, Fichtlscherer S, Zeiher AM and Keller T: Analyzing the release of copeptin from the heart in acute myocardial infarction using a transcoronary gradient model. Sci Rep 6: 20812, 2016.

17. Broessner G, Hasslacher J, Beer R, Lackner P,Lehner GF, Harler U, Schiefecker A, Helbok R, Pfausler B, Hammerer-Lercher A and Joannidis M: Outcome prediction and temperature dependency of MR-proANP and copeptin in comatose resuscitated patients. Resuscitation 89: 75-80, 2015.

18. Schrimpf C, Gillmann HJ, Sahlmann B, Meinders A, Larmann J, Wilhelmi M, Aper T, Rustum S, Lichtinghagen R, Theilmeier G and Teebken OE: Renal function interferes with copeptin in prediction of major adverse cardiac events in patients undergoing vascular surgery. PLoS One 10: e0123093, 2015.
19. Baumert M, Surmiak P, Wiecek A and Walencka Z: Serum NGAL and copeptin levels as predictors of acute kidney injury in asphyxiated neonates. Clin Exp Nephrol 21: 658-664, 2017.

20. Palazzuoli A, Ruocco G, Pellegrini M, Martini S, Del Castillo G, Beltrami M, Franci B, Lucani B and Nuti R: Patients with cardiorenal syndrome revealed increased neurohormonal activity, tubular and myocardial damage compared to heart failure patients with preserved renal function. Cardiorenal Med 4: 257-268, 2014

21. Beltrami M, Ruocco G, Ibrahim A, Lucani B, Franci B, Nuti R and Palazzuoli A: Different trajectories and significance of B-type natriuretic peptide, congestion and acute kidney injury in patients with heart failure. Intern Emerg Med 12: 593-603, 2017.

22. Santos-Araújo C, Leite-Moreira A and Pestana M: Clinical value of natriuretic peptides in chronic kidney disease. Nefrologia 35: $227-233,2015$. 